\title{
Electronic comparison of InAs wurtzite and zincblende phases using nanowire transistors
}

\author{
A.R. Ullah ${ }^{1}$, H.J. Joyce ${ }^{2}$, A.M. Burke ${ }^{1}$, H.H. Tan², C. Jagadish², A.P. Micolich ${ }^{\star}, 1$ \\ ${ }^{1}$ School of Physics, University of New South Wales, Sydney NSW 2052, Australia \\ ${ }^{2}$ Department of Electronic Materials Engineering, Research School of Physics and Engineering, The Australian National University, \\ Canberra ACT 0200, Australia
}

Received XXXX, revised XXXX, accepted XXXX

Published online XXXX

Key words: InAs, nanowire FETs, wurtzite, zincblende.

* Corresponding author: e-mail adam.micolich@nanoelectronics.physics.unsw.edu.au, Phone: +61 293856132

We compare the electronic characteristics of nanowire field-effect transistors made using single pure wurtzite and pure zincblende InAs nanowires with nominally identical diameter. We compare the transfer characterist- ics and field-effect mobility versus temperature for these devices to better understand how differences in InAs phase govern the electronic properties of nanowire transistors.
1 Introduction The small volume and surface area of semiconductor nanowires enables high-quality interfaces that are difficult/impossible in bulk structures [1]. The InAs/InP [2] or Si/Ge [3] heterointerface is the classic example; however, crystal phase homointerfaces in a single semiconductor are also possible. In InAs nanowires these are the zincblende (ZB) phase observed in bulk III-Vs, and the wurtzite (WZ) phase [4]. This phase mixing is usually random and considerable effort has been invested into phase-engineered nanowires [5-8]. One key motivation is using the different band alignments [9-11] to make devices such as quantum dots [12,13]. Another is obtaining phase-pure WZ or ZB nanowires to prevent phase-interface scattering from degrading electrical performance [14, 15].

Studies of how nanowire crystal phase affects electrical properties are at an early stage. Dayeh et al. reported characterization at temperature $T=300 \mathrm{~K}$ of InAs nanowire field-effect transistors (NWFETs) made using pure $\mathrm{ZB}$ nanowires and $\mathrm{WZ}$ nanowires with small ZB segments interspersed axially (approx. $3.5 \mathrm{~nm} \mathrm{ZB}$ per $28.5 \mathrm{~nm} \mathrm{WZ}$ ) [16]. While the mobility $\mu$ was comparable, the ZB NWFETs had higher off-current giving a poor on-off ratio $I_{\text {on }} / I_{\text {off }} \sim 2$ compared to $10^{4}$ for the WZ NWFETs. The difference was attributed to spontaneous polarization charges at the WZ/ZB interfaces. Schroer et al. studied single nanowires with low $\left(<1 \mu \mathrm{m}^{-1}\right)$ and high $\left(>1 \mathrm{~nm}^{-1}\right)$ defect density segments [17]. The mobility at $T=4.2 \mathrm{~K}$ was $\sim 4 \times$ larger for the defect-free segments; while not a direct comparison between WZ and $\mathrm{ZB}$, it shows the potential mobility gains achievable with phase-pure nanowires. Sladek et al. studied InAs nanowire conductivity for three growth methods: one producing WZ nanowires with high stacking fault density, the other two producing ZB nanowires [18]. The measurements showed that doping, intentional or otherwise, dominates over crystal structure in determining conductivity.

Joyce et al. recently developed a growth method for phase-pure WZ and ZB nanowires (NWs) without limiting diameter choice or requiring dopant addition; control was exerted using temperature and V/III ratio [19]. Conditions for obtaining phase-pure InAs nanowires are now well established [7, 20]. Comparative studies of how phase affects thermal conductivity [21] and optical properties [22, 23] have been reported, as has an electrical study involving phase-pure InAs NWs [15]. Thelander et al. studied resistivity $\rho$ versus phase fraction from WZ NWs with low stacking fault density to ZB NWs with periodic twinning structure. For nanowires grown by metalor- 

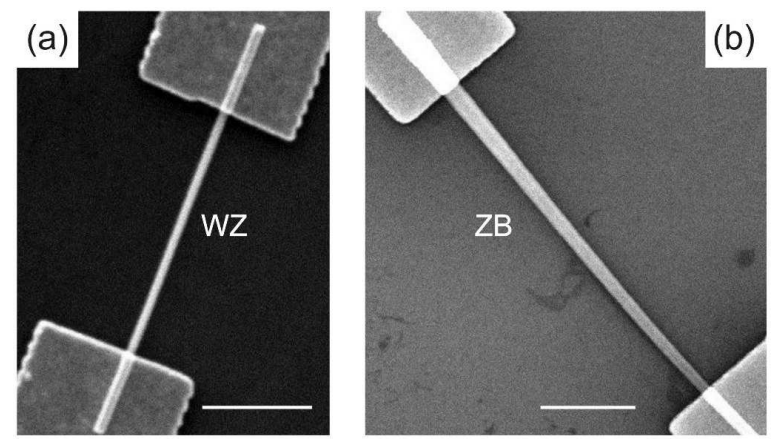

Figure 1 Scanning electron micrograph of completed (a) wurtzite (WZ) and (b) zincblende (ZB) NWFETs. The slight ZB NW taper enables confirmation of the phase species in any given NWFET.

ganic vapor phase epitaxy (MOVPE), phase control was exerted by changing the diameter from 40 (WZ) to $120 \mathrm{~nm}$ (ZB) at fixed $T$ and V/III ratio. WZ NWs were also grown by molecular beam epitaxy (MBE) to account for background doping and diameter effects. The addition of even small amounts of WZ phase to ZB NWs have a significant effect, increasing $\rho$ by two orders of magnitude. Further, pure ZB NWs had $\rho$ comparable to pure WZ NWs, demonstrating that twinning has less effect on $\rho$ than inclusion of extended WZ or ZB segments [15].

Here we report a study of the transfer characteristics and peak field-effect mobility $\mu_{F E}^{p k}$ versus $T$ for NWFETs made using nominally diameter matched pure $\mathrm{WZ}$ and pure ZB InAs nanowires grown by MOVPE using the optimized conditions identified by Joyce et al. [19].

2 Methods InAs NWs were grown by horizontal flow metalorganic chemical vapor deposition (MOCVD) using trimethylindium (TMI) and $\mathrm{AsH}_{3}$. Prior to growth, InAs(111)B substrates were treated with poly-L-Lysine solution followed by a solution of $50 \mathrm{~nm}$ diameter $\mathrm{Au}$ nanoparticles. The substrate was then annealed at $600^{\circ} \mathrm{C}$ in $\mathrm{AsH}_{3}$ to desorb surface contaminants. Growth was performed at $400^{\circ} \mathrm{C}\left(500^{\circ} \mathrm{C}\right)$ for $\mathrm{ZB}$ (WZ) with TMI flow rate $1.2 \times 10^{-5} \mathrm{~mol} / \mathrm{min}$ and $\mathrm{AsH}_{3}$ flow rates $5.5 \times 10^{-4} \mathrm{~mol} / \mathrm{min}\left(3.5 \times 10^{-5} \mathrm{~mol} / \mathrm{min}\right)$. This gives V/III ratios of 46 and 2.9 for $\mathrm{ZB}$ and WZ. Growth was performed for $30 \mathrm{~min}$ at a pressure of 100 mbar with $\mathrm{H}_{2}$ carrier gas added to give $15 \mathrm{slm}$ total flow rate. Our WZ and ZB NWs are exact replicas of those in Figs. 1(d)/2(d) and Figs. 1(a)/2(a) of Ref. [19]. These growths are reproducible, giving pure WZ NWs free of stacking faults and pure ZB NWs without planar crystallographic defects.

NWs were transferred onto a device substrate consisting of a degenerately doped Si wafer capped with $100 \mathrm{~nm}$ $\mathrm{SiO}_{2} / 10 \mathrm{~nm} \mathrm{HfO}_{2}$, and prepatterned with $5 \mathrm{~nm} \mathrm{Ti} / 100 \mathrm{~nm}$ $\mathrm{Au}$ interconnects. The doped substrate serves as the gate. Source and drain contacts were defined by electron beam lithography (EBL). Contact passivation was performed by immersion in $\left(\mathrm{NH}_{4}\right)_{2} \mathrm{~S}_{x}$ solution [24] at $40^{\circ} \mathrm{C}$ for $120 \mathrm{~s}$, which consists of $5 \mathrm{~g} \mathrm{~S}_{2}$ powder added to $52 \mathrm{~mL} 20 \%$ $\left(\mathrm{NH}_{4}\right)_{2} \mathrm{~S}$ solution, diluted 1 : 200 with deionized $\mathrm{H}_{2} \mathrm{O}$ immediately prior to use. $25 \mathrm{~nm} \mathrm{Ni} / 75 \mathrm{~nm}$ Au contacts were deposited by vacuum evaporation immediately thereafter.

Devices were made as 'chips' containing two separate sets of EBL fields. WZ NWs (ZB NWs) were deposited into the first (second) set of EBL fields, enabling parallel fabrication of WZ and ZB NWFETs to ensure common contact properties. The two sets were separated after fabrication and packaged individually in 20-pin ceramic chip carriers. We used scanning electron microscopy (SEM) to confirm a NWFET's phase after electrical measurements were completed; WZ and ZB NWs can be distinguished by taper (see Fig. 1). Our key conclusions have been corroborated in multiple NWFETs from more than one chip.

Low $T$ electrical measurements were performed using a 'dipstick' in a liquid He dewar. We monitored $T$ using a Cernox resistor, with $T=4.2 \mathrm{~K}$ achieved by immersion in liquid and $T>5 \mathrm{~K}$ attained using the temperature gradient of the He dewar atmosphere. The NWFET channel current $I$ was measured using a.c. lock-in techniques with a source-drain voltage $V_{d s}=10(\S 3.1)$ or $4 \mathrm{mV}(\S 3.2)$ at $73 \mathrm{~Hz}$. The gate voltage $V_{g}$ was supplied by a Keithley 2400 enabling continuous gate leakage current $I_{g}$ monitoring, with $I_{g}<10 \mathrm{nA}$ during all measurements.

\section{Results and Discussion}

3.1 Comparison of ZB and WZ NWFET transfer characteristics Figure 2 shows the $I$ versus $V_{g}$ (transfer) characteristics of two ZB and two WZ InAs NWFETs measured in air at $T=300 \mathrm{~K}$, and in He at $T=265$ and $75 \mathrm{~K}$. The WZ NWFETs show consistent characteristics; we obtain $I_{\text {on }} / I_{\text {off }} \sim 2000$ and threshold voltage $V_{\text {th }} \sim 0 \mathrm{~V}$ for WZ-A, WZ-B and several other devices on the same chip (not shown). Atmospheric composition and $T$ both have comparatively weak effects on $V_{\text {th }}$. In contrast, the $\mathrm{ZB}$ NWFET characteristics are more variable, with $V_{\text {th }}$ changing by several volts between devices on the same chip under common conditions, as well as with atmosphere and $T$ for a given NWFET.

One aspect notably different from previous studies is the subthreshold behaviour. We always obtain $I_{\text {on }} / I_{\text {off }}>$ $10^{2}$ for our ZB NWFETs; the on-off ratio for our WZ NWFETs generally tends to be slightly higher than for our ZB NWFETs. This is in contrast to the $I_{\text {on }} / I_{\text {off }}<2$ for ZB InAs NWFETs in Ref. [16]. Dayeh et al. attribute the poorer subthreshold characteristics in their ZB NWFETs to electron accumulation caused by positive surface-state charge. Our data in Figs. 2(c/d) supports the conclusion that the ZB NWFET characteristics are more heavily influenced by surface effects, with greater variations observed in the off-current between air and He atmospheres than for the WZ NWFETs (Fig. 2(a/b)). One explanation is that ZB NWs contain micro-facets with different Miller indices, unlike WZ NWs [19,25]. In addition to an increased den- 

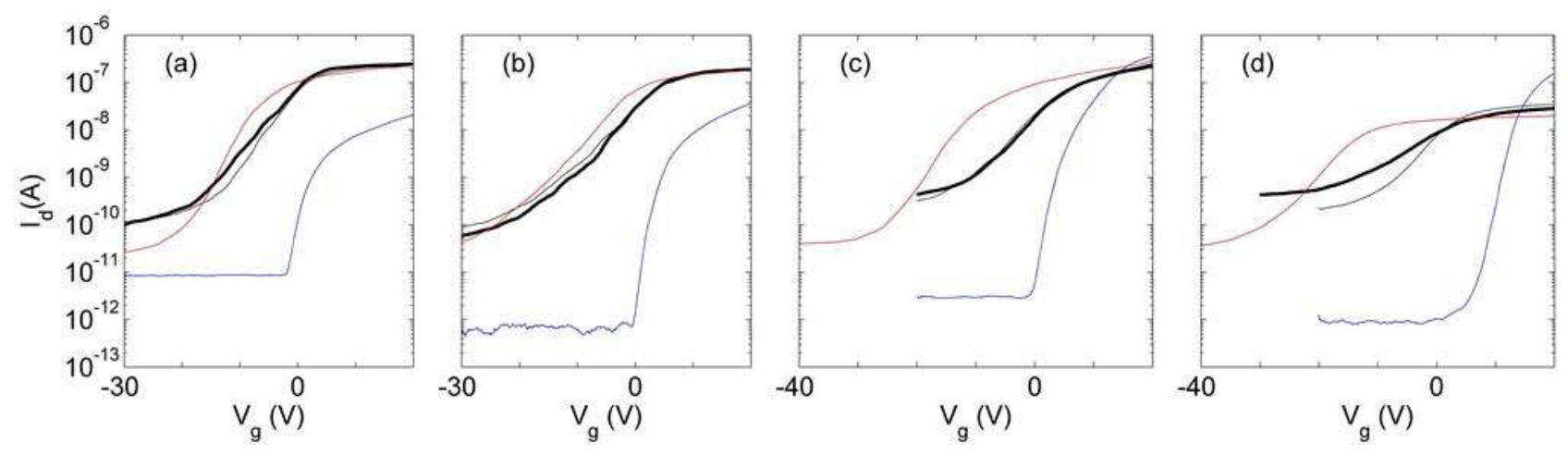

Figure 2 NWFET channel current $I$ versus gate voltage $V_{g}$ at $V_{s d}=10 \mathrm{mV}$ for devices (a) WZ-A, (b) WZ-B, (c) ZB-A and (d) ZB-B. Data obtained in the order: $T=300 \mathrm{~K} /$ air (thin black), $T=75 \mathrm{~K} / \mathrm{He}$ (blue), $T=265 \mathrm{~K} / \mathrm{He}$ (red) and $T=300 \mathrm{~K} /$ air (thick black).

sity of surface states, this may result in differences in In/As surface ratio [25], surface chemistry and surface-state energy spectrum; surface orientation is known to affect surface states and gate behaviour for GaAs, for example [26].

Dayeh et al. also propose that the improved subthreshold characteristics for WZ NWFETs arise from stacking faults, with polarization charge at WZ/ZB stacking fault interfaces giving rise to an axial 'sawtooth' potential that 'cuts through' the channel accumulated by the positive surface charge to ensure complete depletion [16]. One would thus expect the on-off ratio for pure WZ nanowires (i.e., without stacking faults) to be poor. We instead obtain high on-off ratios for our pure WZ NWFETs (Fig. 2(a/b)), which suggests that the off-current difference between WZ and ZB NWFETs goes beyond the spontaneous polarization charge mechanism proposed in Ref. [16]. Instead, it might simply arise from surface-state density/spectrum differences between WZ and ZB NWs. Note well, we do not claim stacking fault interface polarization charge provides no improvement in subthreshold characteristics at all; heterostructure barriers improve NWFET characteristics [27] and there is no reason to expect a WZ/ZB barrier would not do likewise [7]. We only suggest that spontaneous polarization charge is not necessary for high on-off ratio in WZ InAs nanowires.

3.2 Electrical Mobility versus Temperature for WZ and ZB InAs NWFETs To further characterize the transport differences between $\mathrm{ZB}$ and WZ NWs, we obtained transfer characteristics at various $4<T<200 \mathrm{~K}$ for devices WZ-C, WZ-D, ZB-C and ZB-D. The fieldeffect mobility was obtained as $\mu_{F E}=g_{m} L_{G}^{2} / C V_{D S}$, where $g_{m}=\partial I_{D S} / \partial V_{G S}$ is the transconductance, $L_{G}$ is the channel length and $V_{D S}=4 \mathrm{mV}$. The capacitance $C$ was estimated using a cylinder-on-plane model [28]. We obtain both $L_{G}$ and the NW diameter $d$ by SEM after electrical measurements are completed; for the ZB NWs, we assume linear taper and take $d$ as the average of the diameters immediately adjacent to the source and drain contacts. We deal with the $100 \mathrm{~nm} \mathrm{SiO} 2 / 10 \mathrm{~nm} \mathrm{HfO}_{2}$ insu-
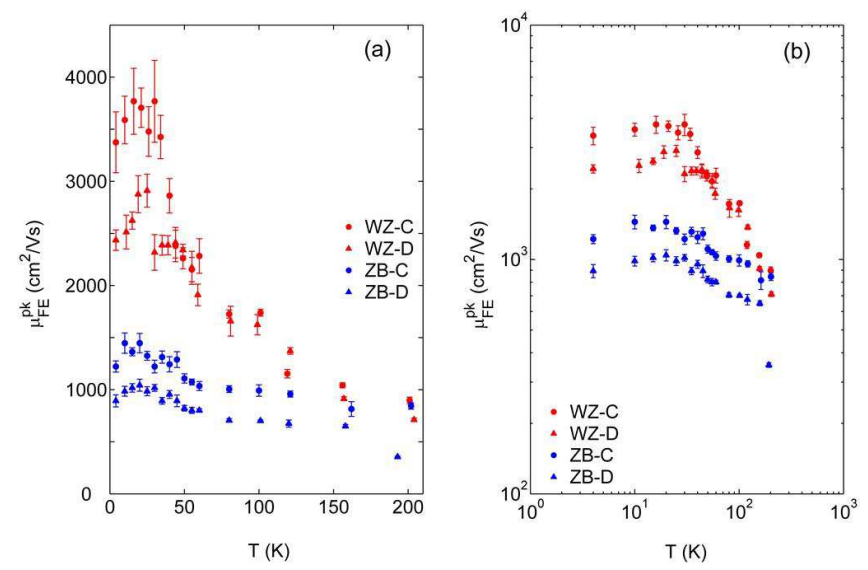

Figure 3 Peak field-effect mobility $\mu_{F E}^{p k}$ vs temperature $T$ on (a) linear and (b) log-log axes for devices WZ-C (red circles), WZ-D (red triangles), ZB-C (blue circles) and ZBD (blue triangles). Data obtained with $V_{s d}=4 \mathrm{mV}$.

lator by assuming a $110 \mathrm{~nm}$ layer with dielectric constant $\epsilon_{\mathrm{eff}}=4.22$.

In Fig. 3(a/b) we plot the peak field-effect mobility $\mu_{F E}^{p k}$ vs $T$ on linear and log-log scales for clarity. For both $\mathrm{WZ}$ and $\mathrm{ZB}$ there is a peak in $\mu_{F E}^{p k}$ versus $T$ at $T=20-30 \mathrm{~K}$, we attribute the low and high $T$ dropoffs in $\mu_{F E}^{p k}$ to ionized impurity and phonon scattering, as per bulk InAs [29,30]. At low $T, \mu_{F E}^{p k}$ is $2-4 \times$ higher for $\mathrm{WZ}$ than $\mathrm{ZB}$; the $\mu_{F E}^{p k}$ values converge as $T \rightarrow 200 \mathrm{~K}$. This is consistent with the similar mobilities obtained for ZB and WZ NWs at $T=300 \mathrm{~K}$ [16], and with the finding that WZ and ZB NWs have relatively similar phonon spectra [21]. The higher low $T \mu_{F E}^{p k}$ for $\mathrm{WZ}$ is not surprising; the lower growth temperature for our ZB NWs will give a higher carbon background impurity level [31] and therefore increased ionized impurity scattering. Surface roughness scattering may also be partially responsible for the re- 
duced low $T \mu_{F E}^{p k}$ for ZB NWs in Fig. 3(a/b), owing to the micro-faceting discussed earlier [19]. Commenting briefly on the functional relationships between $\mu_{F E}^{p k}$ and $T$, one expects $\mu_{F E}^{p k} \propto T^{3 / 2}$ and $T^{-3 / 2}$ in the low and high $T$ limits [29,30]. We find $\mu_{F E}^{p k} \propto T^{0.08}$ for both in the low $T$ limit and $T^{-0.8}$ and $T^{-0.3}$ for $\mathrm{WZ}$ and $\mathrm{ZB}$, respectively, in the high $T$ limit. These differ significantly from expectations for bulk materials; NW-specific calculations of these exponents are not available, but would be an interesting contribution.

4 Conclusions We made a comparative study of NWFETs made using pure WZ and pure ZB InAs NWs with nominally identical diameter grown using MOCVD [19] to establish how the growth differences influence device performance. The WZ NWFETs show more consistent subthreshold characteristics than the ZB NWFETs, and most notably, the ZB NWFET on-off ratio and threshold voltage is more sensitive to temperature and atmospheric composition. This points to surface states playing a greater role in the electronic performance of ZB NWFETs. This may be due to ZB NW surface microfaceting [19]. We find on-off ratios $\sim 100$ for the $\mathrm{ZB}$ NWs, $50 \times$ greater than previously reported [16]. We also found that high on-off ratios persist in WZ NWFETs without stacking faults, demonstrating that WZ/ZB interface polarization charge [16] is not a necessary condition for good subthreshold characteristics. The two phases have similar peak field-effect mobilities in the high temperature limit, consistent with earlier work [16], but the WZ peak mobility is $2-4 \times$ higher in the low $T$ limit, likely due to a combination of higher background impurity levels [31] and increased surface roughness scattering due to micro-faceting in the ZB NWs.

Acknowledgements This work was funded by the Australian Research Council. We thank K. Storm and L. Samuelson for assistance with device fabrication and M.O. Williams for assistance with preliminary studies. This work was performed in part using the NSW and ACT nodes of the Australian National Fabrication Facility (ANFF).

\section{References}

[1] L. Samuelson, Materials Today, 6(10), 22 (2003).

[2] M. Björk, B. Ohlsson, T. Sass, A. Persson, C. Thelander, M. Magnusson, K. Deppert, L.R. Wallenberg, and L. Samuelson, Appl. Phys. Lett. 80, 1058 (2002).

[3] L.J. Lauhon, M.S. Gudiksen, D. Wang, and C.M. Lieber, Nature 420, 57 (2002).

[4] K. Hiruma, M. Yazawa, T. Katsuyama, K. Ogawa, K. Haraguchi, M. Koguchi, and H. Kakibayashi, J. Appl. Phys. 77, 447 (1995).

[5] R.E. Algra, M.A. Verheijen, M. Borgström, L.-F. Feiner, G. Immink, W.J.P. van Enckefort, E. Vlieg, and E.P.A.M. Bakkers, Nature 456, 369 (2008).

[6] P. Caroff, K.A. Dick, J. Johansson, M.E. Messing, K. Deppert, and L. Samuelson, Nature Nanotech. 4, 50 (2009).
[7] K.A. Dick, C. Thelander, L. Samuelson, and P. Caroff, Nano Lett. 10, 3494 (2010).

[8] P. Caroff, J. Bolinsson, and J. Johansson, IEEE J. Sel. Topics Quantum Electron. 17, 829 (2011).

[9] M. Murayama, and T. Nakayama, Phys. Rev. B 49, 4710 (1994).

[10] Z. Zanolli, F. Fuchs, J. Furthmüller, U. von Barth, and F. Bechstedt, Phys. Rev. B 75, 245121 (2007).

[11] A. De, and C.E. Pryor, Phys. Rev. B 81, 155210 (2010).

[12] D. Spirkoska, J. Arbiol, A. Gustafsson, S. Conesa-Boj, F. Glas, I. Zardo, M. Heigoldt, M.H. Gass, A.L. Bleloch, S. Estrade, M. Kaniber, J. Rossler, F. Peiro, J.R. Morante, G. Abstreiter, L. Samuelson, and A. Fontcuberta i Morral, Phys. Rev. B 80, 245325 (2009).

[13] N. Akopian, G. Patriarche, L. Liu, J.-C. Harmand, and V. Zwiller, Nano Lett. 10, 1198 (2010).

[14] Z. Ikonić, G.P. Srivastava, and J.C. Inkson, Phys. Rev. B 48, 17181 (1993).

[15] C. Thelander, P. Caroff, S. Plissard, A.W. Dey, and K.A. Dick, Nano Lett. 11, 2424 (2011).

[16] S.A. Dayeh, D. Susac, K.L. Kavanagh, E.T. Yu, and D. Wang, Adv. Funct. Mater. 19, 2102 (2009).

[17] M.D. Schroer, and J.R. Petta, Nano Lett. 10, 1618 (2010).

[18] K. Sladek, A. Winden, S. Wirths, K. Weis, C. Blömers, Ö. Gül, T. Grap, S. Lenk, M. von der Ahe, T.E. Weirich, H. Hardtdegen, M.I. Lepsa, A. Lysov, Z.-A. Li, W. Prost, F.-J. Tegude, H. Lüth, T. Schäpers, and D. Grützmacher, Phys. Stat. Sol. C 9, 230 (2012).

[19] H.J. Joyce, J. Wong-Leung, Q. Gao, H.H. Tan, and C. Jagadish, Nano Lett. 10, 908 (2010).

[20] K.A. Dick, J. Bolinsson, M.E. Messing, S. Lehmann, J. Johansson, and P. Caroff, J. Vac. Sci. Technol. B 29, 04D103 (2011).

[21] F. Zhou, A.L. Moore, J. Bolinsson, A. Persson, L. Fröberg, M.T. Pettes, H. Kong, L. Rabenberg, P. Caroff, D.A. Stewart, N. Mingo, K.A. Dick, L. Samuelson, H. Linke, and L. Shi, Phys. Rev. B 83, 205416 (2011).

[22] M.H. Sun, E.S.P. Leong, A.H. Chin, C.Z. Ning, G.E. Cirlin, Yu.B. Samsonenko, V.G. Dubrovskii, L. Chuang, and C. Chang-Hasnain, Nanotechnol. 21, 335705 (2010).

[23] C. Wilhelm, A. Larrue, X. Dai, D. Migas, and C. Soci, Nanoscale 4, 1446 (2012).

[24] D. Suyatin, C. Thelander, M. Björk, I. Maximov, and L. Samuelson, Nanotechnol. 18, 105307 (2007).

[25] M.H. Sun, H.J. Joyce, Q. Gao, H.H. Tan, C. Jagadish, and C.Z. Ning, Nano Lett. 12, 3378 (2012).

[26] A.M. Burke, D.E.J. Waddington, D.J. Carrad, R.W. Lyttleton, H.H. Tan, P.J. Reece, O. Klochan, A.R. Hamilton, A. Rai, D. Reuter, A.D. Wieck, and A.P. Micolich, Phys. Rev. B 86, 165309 (2012).

[27] L. Fröberg, C. Rehnstedt, C. Thelander, E. Lind, L.-E. Wernersson, and L. Samuelson, IEEE Electron. Dev. Lett. 29, 981 (2008).

[28] S.A. Dayeh, D.P.R. Aplin, X. Zhou, P.K.L. Yu, E.T. Yu, and D. Wang, Small 3, 326 (2007).

[29] E. Conwell, and V. Weisskopf, Phys. Rev. 77, 388 (1950).

[30] D.L. Rode, Phys. Rev. B 8, 3287 (1971).

[31] C. Thelander, K.A. Dick, M.T. Borgström, L.E. Fröberg, P. Caroff, H.A. Nilsson, and L. Samuelson, Nanotechnology 21, 205703 (2010). 